\title{
Priority based Fuzzy Decision Packet Scheduling Algorithm for QOS in Wireless Sensor Network
}

\author{
Varsha Jain \\ M.Tech in Computer Science, \\ GWECA, Ajmer, \\ RTU, Kota
}

\author{
Shweta Agarwal \\ M.Tech in Computer Science, \\ GWECA, Ajmer, \\ RTU, Kota
}

\author{
Kuldeep Goswami \\ Assistant Professor, \\ GWECA, Ajmer, \\ RTU, Kota
}

\begin{abstract}
As the WSN getting involved in multi-objective system it needs to cope up with different types of data and to properly transfer them according to their requirements. Since the different kind of data have different delay and bandwidth requirements constrains which needed to be fulfilled without exceeding the node internal constrains such as queue buffer overflow. Previous studies shown that the packet scheduling algorithm for WSN does not perform effectively and efficiently which result in degraded QoS. There are different ways to packet scheduling such as to allocate overall bandwidth and time slot on request by following strict priority to adaptive priority basis. Because complexity of task some of these scheduling algorithms require complex mathematical approach to solve the issue. This paper present a fuzzy based algorithm to overcome all related problems which are explained earlier other advantage of using fuzzy logic is that it does not require complex mathematical calculations, finally the simulation of the proposed algorithm is performed using NS2 and the results shows that the proposed algorithm satisfactorily fulfill the system requirements.
\end{abstract}

\section{General Terms}

Packet scheduling Algorithm.

\section{Keywords}

Wireless sensor network, packet scheduling, Fuzzy Logic.

\section{INTRODUCTION}

Recent development of low-power analog and digital electronics makes it feasible to design compact and inexpensive battery operated sensor units, equipped with wireless communication and computing capabilities emerges. Due to the continuous decrease in the cost, large number of sensor nodes may be deployed as sensor networks and can be used for scientific research or other applications, such as monitoring the battlefield or for weather forecasting. Providing quality service to users is one of the important issue especially for the recent applications of sensor networks in real time applications. In many applications of WSN bulk data amount is collected by the sensors in a large sensor network and sent back to base station for processing and analysis. However, practically in most of the cases only the fractional amount of raw data from sensor nodes relate to the objective purposes and can be considered as useful or it can be understand that node should only transmit or forward the data needed for application in base station and for such cases the data which has lower importance can be delayed or dropped to provide extra time slot for important data this can reduce the unnecessary data as well as quality of service. In other aspect for real time application, some data has are more valuable and time critical requirements than the others. Also to save power in critical situations (where the node is running out of power) processing of several less important tasks can be replaced by one more important task that will consume less energy. Furthermore the packet scheduling has special importance in multi-objective sensor nodes are used which are capable of monitoring the multiple parameters (for example a sensor may measure the temperature, pressure and simultaneously send images of the environment) where every service type have different usability constrains therefore, the sensor nodes should have proper algorithm to maintain these constrains. Hence the packet-scheduling algorithm has the purpose to meet its working requirement and constraints, so that the performance of the sensor network can be guaranteed. Another constrain of the packet scheduler beside the Energy and Time constraints is packet drop due to timeout or buffer overflow are considered as the third constraint for each task. In this paper, we simply proposed a fuzzy logic based packet scheduling algorithm. The purpose of proposed algorithm is to maximize the system QoS while maintaining the time, energy and packet drop constraints. In rest of the paper chapter two presents a brief review on packet scheduling algorithms while third section discusses the fuzzy logic theory. The fourth section explains the proposed approach follower by simulation results in sixth section. Finally the conclusion and the future scope of the proposed algorithm is discussed in section seven.

\section{LITERATURE REVIEW}

Since the well proven applicability of packet scheduling in quality of service and efficient energy utilization, with addressing critical issues of battery-operated wireless sensor networks; many related researches have been done to address these problems by using different approaches. Shu Lei et al [8] presented the Two Tiers Buffer model (one of sensor networks and other of sensor node) which can be used as platform for the Energy, Time, Reward, and Interest (ETRI) packet scheduling algorithm to schedule the coming packets. By using ETRI principles, the proposed algorithm can dynamically combine these ETRI constraints achieve diverse scheduling characteristics for different sensor nodes working environments and purposes, the algorithm can substantially improve the information quality and average performance of whole sensor networks. A threshold-based medium access control (MAC) scheme for uplink packet transmission for single beam opportunistic communication which exploits multiuser diversity gain without feedback in a decentralized manner is proposed by Hakk Soy et al [7]. In the technique Packet transfer from sensor nodes to the controller node is performed on the basis of channel. The optimum threshold for minimum required SNR is determined to maximize the probability of successful packet transmission where only one sensor node transmits its packet in one time- 
slot. Traffic Scheduling Scheme for Differentiated Services of Healthcare Systems is proposed in [4]. The paper proposes a priority based traffic and scheduling scheme for WBAN (wireless body area networks). It is based on data classification and prioritization according to their current status and diseases. In the proposal urgent data time constrains management are the considered as key issue for queue scheduling and path choice issues and the urgent data are delivered on time to provide a QoS guarantee for WBAN. Shs Liu et al [13] proposed an algorithm to simultaneously achieve the seemingly contradictory goals of energy efficiency and low latency called Convergent MAC (CMAC). CMAC zero communication when there is no traffic to avoid synchronization overhead while supporting low latency it also allows sensor nodes to operate at very low duty cycles. CMAC first uses anycast to wake up forwarding nodes, and then converges gradually from route-suboptimal any cast with unsynchronized duty cycling to route-optimal unicast with synchronized scheduling. Time-Optimum Packet Scheduling for Many-to-One Routing in presented in [11]. In the proposed packet scheduling algorithm, each node goes to sleep whenever it is not transceiving, so that the energy waste of idle listening is also mitigated. The proposed algorithm also achieves time optimum scheduling in heterogeneous scenarios, where each sensor reports a heterogeneous amount of data in each round. The distributed implementation of the algorithm is also implemented.

\section{FUZZY LOGIC}

Fuzzy logic is an approach to computer science that mimics the way a human brain thinks and solves problems [15]. The idea of fuzzy logic is to approximate decision making using natural language terms instead of quantitative terms. It is generally considered as modeling of information where it cannot be defined precisely, but some broad definitions can be formed. Because of its simplicity and effectiveness, Fuzzylogic technology has gained many applications in scientific and industrial applications.

A typical architecture of FLC is shown below, which comprises of four principal comprises: a fuzzifier, a fuzzy rule base, inference engine, and a defuzzifier.

Fuzzifier: Used to transform crisp measured data (e.g. speed is $10 \mathrm{mph}$ ) into suitable linguistic values (i.e. fuzzy sets, for example, speed is too slow).

Fuzzy Rule Base: stores the empirical knowledge of the operation of the process of the domain experts.

Inference Engine: is the kernel of a FLC, and it has the capability of simulating human decision making by performing approximate reasoning to achieve a desired control strategy.

Defuzzifier: is utilized to yield a nonfuzzy decision or control action from an inferred fuzzy control action by the inference engine.

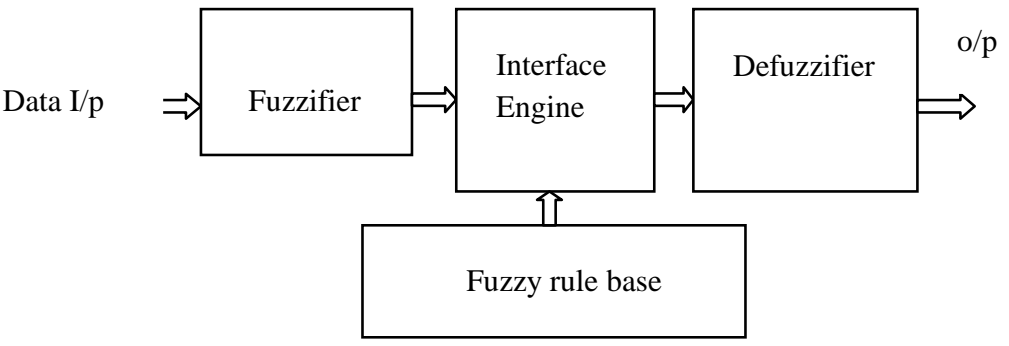

Fig 1: Block Diagram of Fuzzy Controller

\section{PROPOSED ALGORITHM}

This section presents the proposed work and algorithm to overcome all related problems which has detailed in earlier sections. The scheduler uses the fuzzy logic to achieve all goals. The advantage of using fuzzy logic is that it can be designed for complex problems with only logical rules hence does not require complex mathematical calculation.

The proposed algorithm can be written as follows:

\section{Algorithm:}

Start:

1. Sense Available Bandwidth (BW);

2. Sense Traffic Load in the Network;

3. From the input queue check the requested data to be transmitted

4. Check their priority, data size and BWrequirement;

5. Apply fuzzy rule for each entry;

6. Serve the request according to fuzzy output;

7. Stop.

End

Fuzzy Logic Scheduler: used in step 5 of main algorithm

Start:

1. Apply fuzzy logic with priority, data size and $\mathrm{BW}$-required in the following way.

a.) A fuzzy rule in the form "If.... Then";

a.) find membership function;

b.) maintain fuzzy table from fuzzy matrix;

2. Repeat the procedure for all entries.

3. Go to step (6) of main algorithm;

End 


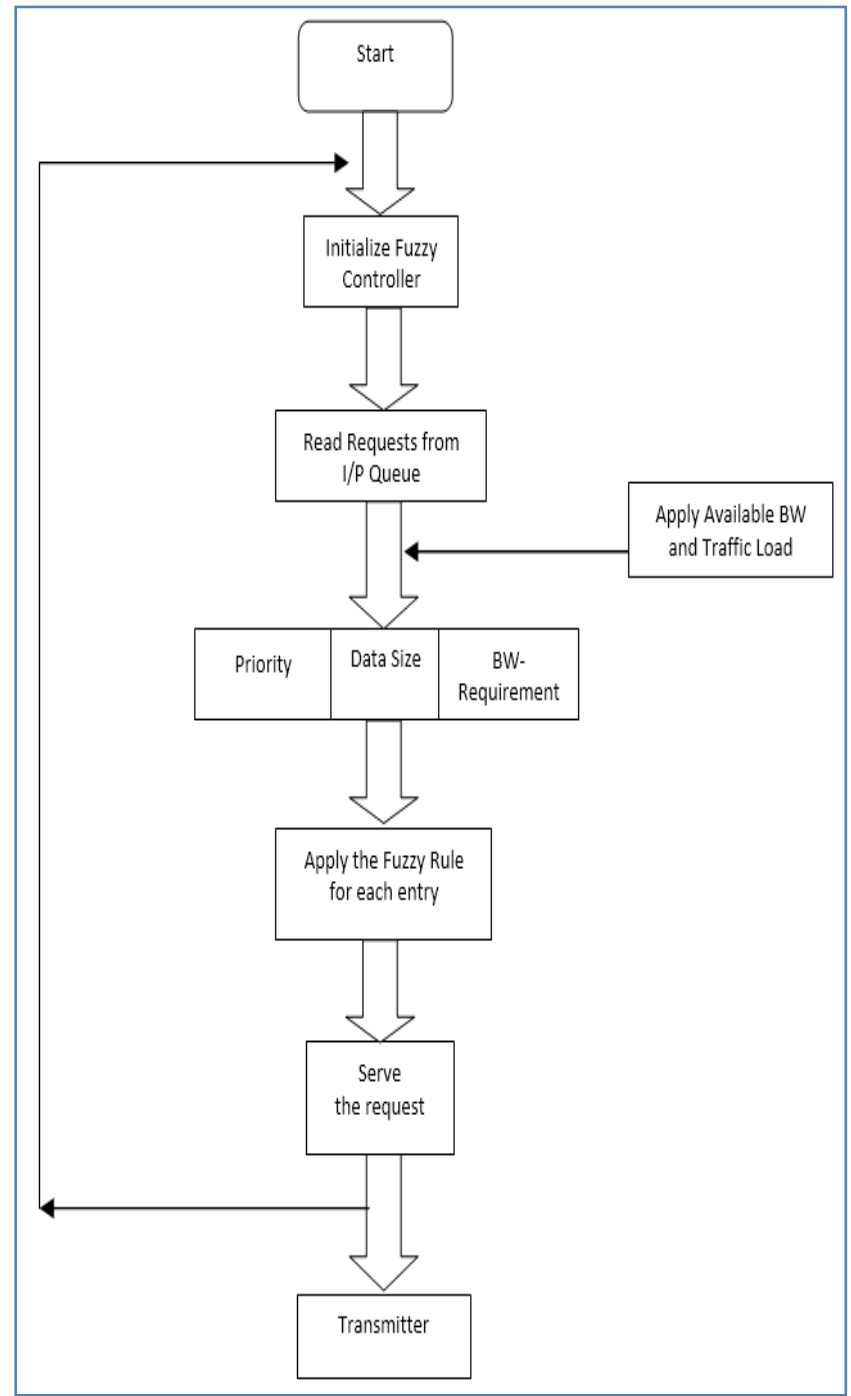

Fig 2: Flow Chart of the proposed Algorithm.

The start is the rounded rectangle box which initializes the Fuzzy Controller by loading the pre-defined rules. Next box is to read the input queue to check the requests in waiting conditions. The according to requests type the value of priority, data size of request and the required BW is estimated. After that the channel and network condition is analyzed. Now the fuzzy rules are applied to each entry. After completing this phase provide the transmission priority to each request according to fuzzy output.

\section{SIMULATION RESULTS}

This section presents the performance evaluation for the proposed scheme. The metrics used herein for the evaluation in terms of QoS are end-to-end delay and packet drop rate and throughput.

The simulation of the system is performed in NS2 with different number of nodes varying from 50 to 100 .

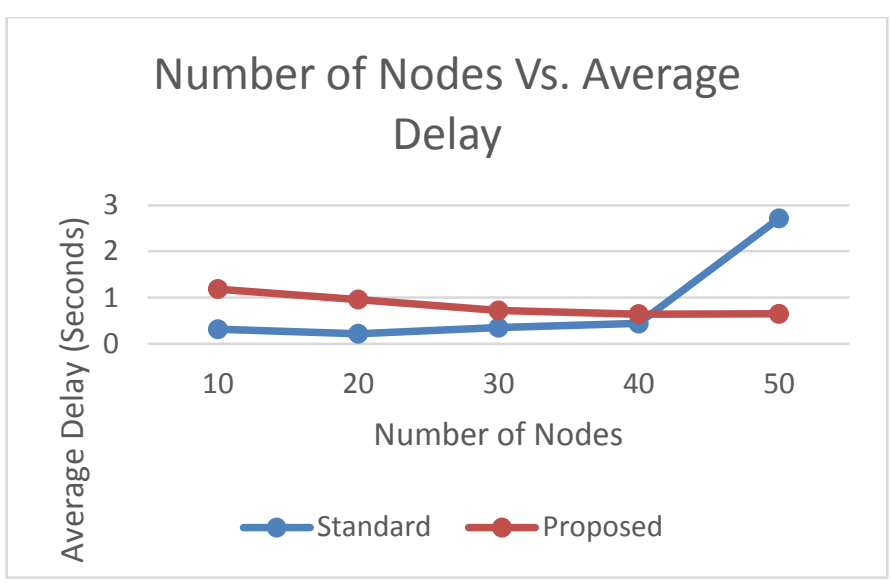

Fig 3: comparison of average end to end delay for different numbers of participating nodes

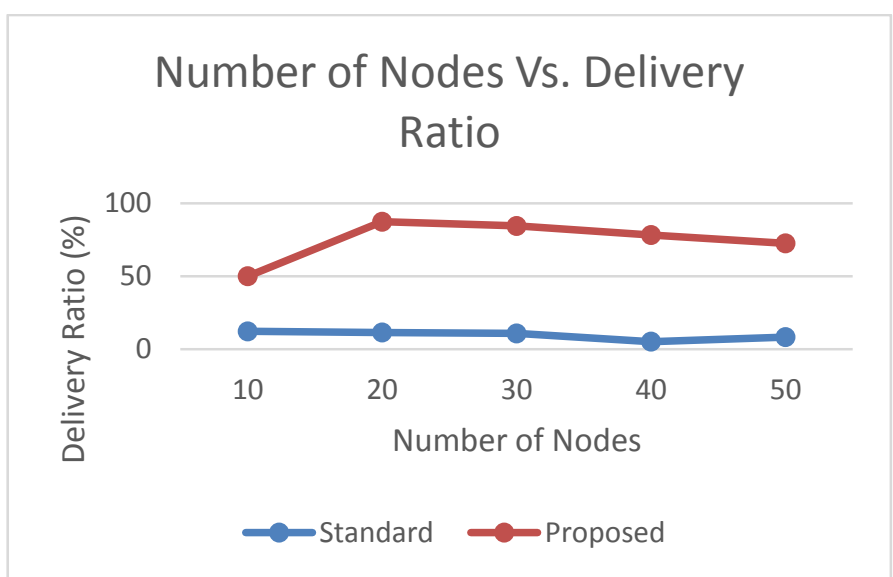

Fig 4: comparison of Delivery Ratio (in Percentage) for different numbers of participating nodes

\section{Number of Nodes Vs. Throughput (Transferred)}

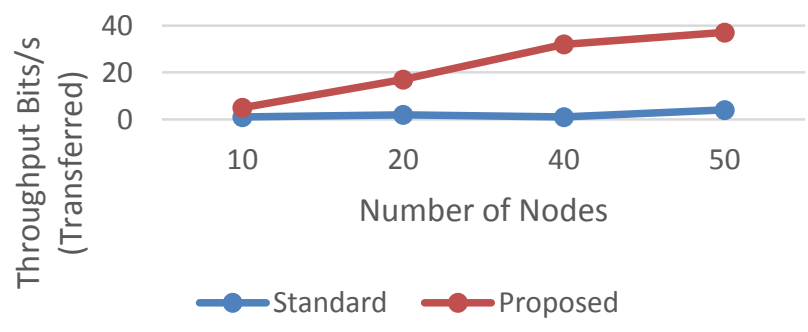

Fig 5: comparison of Throughput for different numbers of participating nodes. 


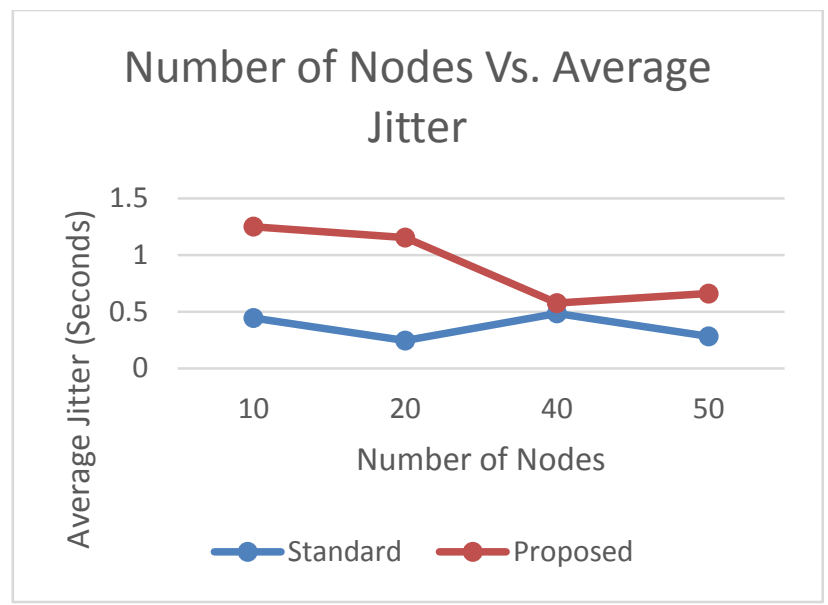

Fig 6: plot of the table data for number of nodes Vs. Average Jitter.

\section{CONCLUSION}

In this paper, we presented the fuzzy logic based packet scheduling scheme. The presented algorithm can be useful for the time, priority and Bandwidth optimum applications in wireless sensor networks with large number of nodes. The implementation and simulation of the proposed algorithm with different node density shows that the proposed algorithm improves the delivery ratio from 40 to 60 at heave node density also the average packet delay and throughput gets increased by acceptable margin. Fortunately, proposed scheduling algorithm works locally hence no communication overhead required and each node calculates its own decision according to gathered information. The achieved results also encourage the application of nonconventional system (fuzzy scheduler) for such applications. Although the further modifications and improvements on the fuzzy rules may be performed in future work.

\section{REFERENCES}

[1] Bo Ji, Changhee Joo, and Ness B. Shroff "Throughputoptimal Scheduling in Multi-hop Wireless Networks without Per-flow Information", Networking, IEEE/ACM Transactions on (Volume:21, Issue: 2 ) April 2013.

[2] Kuo-Feng Huang, Shih-Jung Wu "Real-time-servicebased Distributed Scheduling Scheme for IEEE 802.16j Networks", JOURNAL OF NETWORKS, VOL. 8, NO. 3, MARCH 2013.

[3] Cecile Kateretse, Ga-Won Lee, Eui-Nam Huh "A Practical Traffic Scheduling Scheme for Differentiated Services of Healthcare Systems on Wireless Sensor Networks", Wireless Personal Communications July 2013, Volume 71, Issue 2, pp 909-927, Springer.

[4] Jithin Jagannath, Anu Saji, Hovannes Kulhandjian, Yifan Sun, Emrecan Demirors, and Tommaso Melodia "A Hybrid MAC Protocol with Channel-dependent Optimized Scheduling for Clustered Underwater Acoustic Sensor Networks", WUWNet '13 Proceedings of the Eighth ACM International Conference on Underwater Networks and Systems Article No.3.
[5] Bo Ji, Changhee Joo and Ness B. Shroff "Delay-Based Back-Pressure Scheduling in Multihop Wireless Networks", INFOCOM, 2011 Proceedings IEEE Date of Conference: 10-15 April 2011.

[6] Nidal Nasser, Lutful Karim, and Tarik Taleb "Dynamic Multilevel Priority Packet Scheduling Scheme for Wireless Sensor Network", IEEE Transactions on Wireless Communications, Vol. 12, No. 4, April 2013.

[7] Hakkı Soy, Özgür Özdemir, Mehmet Bayrak, Ridha Hamila, Naofal Al-Dhahir "Decentralized multiuser diversity with opportunistic packet transmission in MIMO wireless sensor networks", AEU - International Journal of Electronics and Communications Volume 67, Issue 11, November 2013, Pages 910-925, ScienceDirect.

[8] Shu Lei, S.Y. Lee, Yang Jie, Kyung Hee "ETRI: A Dynamic Packet Scheduling Algorithm for Wireless Sensor Networks", Proceedings of ETRSI, 2004.

[9] C. Jandaeng, W. Suntiamontut, N. Elz "PSA: The Packet Scheduling Algorithm for Wireless Sensor Networks", International journal on applications of graph theory in wireless ad hoc networks and sensor networks (GRAPHHOC) Vol.3, No.3, September 2011.

[10] Busola S. Olagbegi and Natarajan Meghanathan "A Review of The Energy Efficient and Secure Multicast Routing Protocols for Mobile Ad Hoc Networks", nternational journal on applications of graph theory in wireless ad hoc networks and sensor networks (GRAPHHOC) Vol.2, No.2, June 2010.

[11] Wen-Zhan Song, Fenghua Yuan, Richard LaHusen "Time-Optimum Packet Scheduling for Many-to-One Routing in Wireless Sensor Networks", Mobile Adhoc and Sensor Systems (MASS), 2006 IEEE International Conference on Oct. 2006.

[12] Sinem Coleri Ergen and Pravin Varaiya "TDMA scheduling algorithms for wireless sensor networks", Wireless NetworksThe Journal of Mobile Communication, Computation and Information, 27 May 2009, Springer.

[13] Sha Liu, Kai-Wei Fan and Prasun Sinha "CMAC: An Energy Efficient MAC Layer Protocol Using Convergent Packet Forwarding for Wireless Sensor Networks", Sensor, Mesh and Ad Hoc Communications and Networks, 2007. SECON '07. 4th Annual IEEE Communications Society Conference on 18-21 June 2007.

[14] Chonggang Wang, Kazem Sohraby, Victor Lawrence, Bo Li, Yueming Hu "Priority-based Congestion Control in Wireless Sensor Networks", Sensor Networks, Ubiquitous, and Trustworthy Computing, 2006. IEEE International Conference on (Volume:1 ), 5-7 June 2006.

[15] Bih, J. "Paradigm shift an introduction to fuzzy logic", Potentials, IEEE (Volume:25 , Issue: 1 ) Jan.-Feb. 2006. 